\title{
Current trends in physiological sciences: from cell signals to the biology of aging
}

\author{
Guest Editors \\ V.L. Jakovljevic \\ University of Kragujevac, Kragujevac, Serbia \\ D.M. Djuric \\ University of Belgrade, Belgrade, Serbia \\ D. Jacques \\ University of Sherbrooke, Sherbrooke, Quebec, Canada
}

